\title{
Retracted: Role of LncRNA MALAT-1 on Induced Submandibular Gland Carcinoma in Albino Rats Treated with Induced Pluripotent Stem Cells (Histological and Immunhistochemical Study)
}

\author{
Eman Mohamed Faruk ${ }^{1 *}$, Sherifa Abd El-Salam ${ }^{1}$, Dina Sabry ${ }^{2}$, Engy Medhat ${ }^{2}$ \\ and Yasmine Alaa EI-Din ${ }^{3}$ \\ ${ }^{1}$ Departments of Histology and Cytology, Faculty of Medicine, Benha University, Benha, Egypt. \\ ${ }^{2}$ Department of Medical Biochemistry and Molecular Biology, Faculty of Medicine, Cairo University, \\ ${ }^{3}$ Department of Oral Pathology, October 6 University, Giza, Egypt.
}

Authors' contributions

This work was carried out in collaboration among all authors. All authors read and approved the final manuscript.

Article Information

DOI: $10.9734 / A R R B / 2019 / v 32 i 130072$ Editor(s): (1) Dr. George Perry, Dean and Professor of Biology, University of Texas at San Antonio, USA.
Reviewers: (1) Dr. Niranjan Kc, Shri Dharmasthala Manjunatheshwara University, India. (2) Nisheeth Saawarn, Bhabha University, India. Complete Peer review History: http://www.sdiarticle3.com/review-history/49452

Salivary gland tumors show complex histopathology and the treatment depends mainly on the stage of cancer. Induced pluripotent stem cells (iPS) have a great role in regenerative medicine as they can generate pluripotent stem cells from any available cell types as fibroblast. Thus, the aim of this work is to investigate the possible therapeutic effect of (iPS) on induced salivary gland cancer through evaluation of the silent information regulators of sirtuin-1 (Sirt-1), Tgf- $\beta$ genes and their protein expressions in addition to LncRNA MALAT-1 expression. Thirty male albino rats were employed and divided into three groups (ten rats for each group), group 1 (control): Rats were injected with phosphate buffered saline (PBS), group II induced squamous cell carcinoma (SCC): rats were injected with squamous carcinoma cells (SCC), group III (induced SCC/iPS): SCC treated rats treated with $5 \times 10^{6}$ iPS cells. Submandibular specimens were taken and prepared for 
histological, histochemical and immunohistochemical studies for Bax and TGF- $\beta 3$ protein. Also, Real time PCR was performed for Sirt-1, Tgf- $\beta$, and MALAT-1 LncRNA genes expression. SIRT-1 and TGF- $\beta$ protein level expression was assessed by western blot technique. Group III (iPS treated group) revealed more or less normal acinar structure with normal rearrangement of acini and normal intralobular ducts with an increase in their number. In the iPS treated group there was increasing in the amount of mucopoly saccharide in the acinar cells and intensity of BAX immunostaining while, TGF- $\beta 3$ was decreased in its intensity in comparison to that of the cancer treated group. In addition to Sirt-1, Tgf- $\beta$, and MALAT-1 LncRNA expressions were increased in cancer group compared to iPS treated and control groups. Induced pluripotent stem cells play a potential therapeutic role in treatment of induced submandibular gland carcinoma. 